\title{
High Resolution Signal Averaged Electrocardiography May Improve Diagnosis of Atrial Fibrillation and Reduce Stroke
}

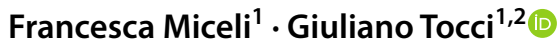

Received: 10 May 2020 / Accepted: 30 May 2020 / Published online: 6 June 2020

(C) Italian Society of Hypertension 2020

Keywords Atrial fibrillation $\cdot \mathrm{p}$ wave duration $\cdot \mathrm{p}$ wave morphology $\cdot \mathrm{p}$ wave axis $\cdot \mathrm{p}$ wave analysis $\cdot$ High resolution signal averaged electrocardiography $\cdot$ Stroke

Over the last decades, our knowledge concerning anatomic, neurohormonal and electrical abnormalities that may trigger atrial fibrillation (AF) has markedly evolved. Despite these improvements, AF still represents the most common cardiac arrhythmia in the clinical practice, and remains one of the major cause of stroke worldwide [1].

Pathophysiology of AF is multifactorial, by linking electrical alterations with neurohormonal factors and structural changes of the left atrium (LA). Also pulmonary veins have a central role in the pathophysiology of AF, through reentry circuits that, spreading to the atria, may generate a "chaotic" electrical activation. Structural alterations, such as LA enlargement, fibrosis and adipose tissue deposition, may further promote a disorganized electrical conduction, by creating anatomical conduction blocks, which generate and sustain the development of AF.

Up to date, the clinical presentation of AF is heterogenous and remains a challenging field of study.

Since up to $20 \%$ of AF patients are asymptomatic and one third of stroke are crytogenic (namely, unexplained), early clinical or biochemical markers able to identify patients atrisk of having AF are needed [2]. In this setting, 12-lead electrocardiogram (ECG) is a simple, repeatable and costeffectiveness method to evaluate markers of AF. Indeed conventional ECG allows to analyse $\mathrm{p}$ wave duration (PWD), $p$ wave morphology and $p$ wave axis, the so called " $p$ wave analysis".

Giuliano Tocci

giuliano.tocci@uniroma1.it

1 Hypertension Unit, Division of Cardiology, Department of Clinical and Molecular Medicine, Faculty of Medicine and Psychology, University of Rome Sapienza, Sant'Andrea Hospital, Rome, Italy

2 IRCCS Neuromed, Pozzilli (IS), Italy
In this issue of High Blood Pressure and Cardiovascular Prevention, Francia and coworkers reported a comprehensive review on the main ECG markers for early AF detection in asymptomatic patients, with a particular focus on PWD and $\mathrm{p}$ wave morphology (reference to be added). Many studies reported a strong correlation between PWD and the occurrence of $\mathrm{AF}$, by confirming the linear correlation between PWD and the risk of AF [3]. Other studies showed that $p$ wave morphology may be influenced not only by electrical atrial remodelling, but also by structural changes, such as LA dilation and hypertrophy $[4,5]$. However, this latter parameter has demonstrated to provide relatively low specificity for the prediction of LA enlargement. Finally, the $p$ wave axis, was recently considered as a predictive factor of AF-related ischemic stroke [6].

Although standard ECG is widely used to evaluate $p$ wave parameters, there are some limitations due to its relatively low accuracy to detect low electrical atrial potentials. In order to overcome this issue, high resolution signal averaged electrocardiography (SAECG) has been adopted for $\mathrm{p}$ wave analysis. Indeed, a study showed that filtered PWD was significantly prolonged in AF patients compared to non-AF individuals [7]. Moreover, patients with cryptogenic stroke and paroxysmal AF, showed a longer PWD on SAECG with a positive predictive value [8]. SAECG was also used to evaluate $\mathrm{p}$ wave analysis in different clinical presentations, such as in those patients affected by hypertrophic cardiomyopathy, in whom a prolonged PWD resulted to be correlated to LA enlargement.

Particular interest was given to the therapeutic implications of SAECG in evaluating the risk of AF recurrence. Although there are different types of pacing (e.g. bi-atrial, Bachmann's Bundle pacing), no evidence suggests a definite atrial pacing algorithms to prevent AF occurrence. On the other hand, there is a clear correlation between reduced 
incidence of AF recurrence and reduced PWD, thus demonstrating a predictive role of PWD in reducing AF events. Another therapeutic option for AF is the ablation, and several evidence demonstrated that PWD changes before and after AF ablation may be associated with AF relapses [9]. Some studies have also compared the accuracy between standard ECG and SAECG, by confirming that SAECG is a more reliable tool to assess PWD.

In line with the available evidence, 12-lead ECG still represents the first-line diagnostic approach in patients with AF or at risk of having $\mathrm{AF}$, since it may be considered a simple, repeatable and cost-effectiveness method to analyse early ECG markers of AF, including left ventricular remodelling and hypertrophy, in asymptomatic patients in a setting of clinical practice [10]. To overcome the intrinsic limitations of this method, SAECG can be effectively and widely used to improve diagnostic accuracy. This review emphasises the role of $p$ wave analysis as a suitable tool to early identify patients at risk of AF and, as a consequence, to provide useful marker for choosing the appropriate therapeutic strategy, by improving clinical outcomes in those patients at high risk of AF-related stroke.

\section{References}

1. Kirchhof P, Benussi S, Kotecha D, Ahlsson A, et al. 2016 ESC guidelines for management of atrial fibrillation developed in collaboration with EACTS. Eur Heart J. 2016;37(38):2893-962.
2. Xiong Q, Proietti M, Senoo K, Lip GY. Asymptomatic versus symptomatic atrial fibrillation: a systematic review of age/ gender differences and cardiovascular outcomes. Int J Cardiol. 2015;191:172-7.

3. Francia P, et al. P-wave duration in lead aVR and the risk of atrial fibrillation in hypertension. Ann Noninvasive Electrocardiol. 2015;20(2):167-74.

4. Andlauer R, Seemann G, Baron L, Dössel O, Kohl P, Platonov $\mathrm{P}$, Loewe A. Influence of left atrial size on P-wave morphology: differential effects of dilation and hypertrophy. Europace. 2018;20(suppl 3):iii36-iii44.

5. Francia P, Ricotta A, Balla C, Adduci C, Semprini L, Frattari A, Modestino A, Mercanti F, Sensini I, Caprinozzi M, Tocci G, Volpe M. P-wave duration in lead aVR and the risk of atrial fibrillation in hypertension. Ann Noninvasive Electrocardiol. 2015;20(2):167-74.

6. Maheshwari A, Norby FL, Roetker NS, Soliman EZ, Koene RJ, Rooney MR, O'Neal WT, Shah AM, Claggett BL, Solomon SD, Alonso A, Gottesman RF, Heckbert SR, Chen LY. Refining prediction of atrial fibrillation-related stroke using the P2-CHA2DS2VASc Score. Circulation. 2019;139(2):180-91.

7. Steinberg JS, Zelenkofske S, Wong SC, Gelernt M, Sciacca R, Menchavez E. Value of the P-wave signal-averaged ECG for predicting atrial fibrillation after cardiac surgery. Circulation. 1993;88(6):2618-22.

8. Gencel L, Poquet F, Gosse P, Haissaguerre M, Marcus FI, Clementy J. Correlation of signal-averaged $\mathrm{P}$ wave with electrophysiological testing for atrial vulnerability in strokes of unexplained etiology. Pacing Clin Electrophysiol. 1994;17(11 Pt 2):2118-244.

9. Pranata R, Yonas E, Vania R. Prolonged P-wave duration in sinus rhythm preablation is associated with atrial fibrillation recurrence after pulmonary vein isolation. A systematic review and metaanalysis. Ann Noninvasive Electrocardiol. 2019;24(5):e12653.

10. Miceli F, et al. Conventional and new electrocardiographic criteria for hypertension-mediated cardiac organ damage: a narrative review. J Clin Hypertens (Greenwich). 2019;21(12):1863-71. 